\title{
Descriptive analysis and early-stage consumer acceptance of yogurts fermented with carrot juice
}

\author{
M. A. Cliff, ${ }^{* 1}$ L. Fan, $\dagger$ K. Sanford, $†$ K. Stanich, ${ }^{*}$ C. Doucette, $\nmid$ and N. Raymond $\dagger$ \\ ${ }^{*}$ Pacific Agri-Food Research Centre, Summerland, BC, VOH 1Z0, Canada \\ †Atlantic Food and Horticulture Research Centre, Kentville, NS, B4N 1J5, Canada
}

\section{ABSTRACT}

This research explored the sensory characteristics and consumer acceptance of novel probiotic unsweetened yogurts. Yogurts were made with 4 carrot juice levels (8, 16, 24, and 32\%), 2 firmness levels (regular, 45 $\mathrm{g} / \mathrm{L}$ milk solids; firm, $90 \mathrm{~g} / \mathrm{L}$ milk solids), and 2 starter cultures (C1, C2). The sensory profile characterized the color intensity (before and after stirring), carrot flavor, sourness, and 7 texture/mouth-feel attributes (astringency, chalkiness, mouth-coating, thickness, smoothness, creaminess, and graininess). The influence of carrot juice level and firmness level were evaluated using ANOVA, polynomial contrasts, and principal component analysis. Mean scores and standard errors were calculated. Consumer acceptance panels in Wolfville, Nova Scotia $(\mathrm{n}=56)$, and in Vancouver, British Columbia (Asian $\mathrm{n}=72$, non-Asian $\mathrm{n}=72$ ), evaluated the hedonic responses to the $\mathrm{C} 1$ and $\mathrm{C} 2$ formulations, respectively. We observed increases in color intensity, carrot flavor, creaminess, mouth-coating, and chalkiness with increasing carrot juice levels, as well as increases in color intensity, carrot flavor, creaminess, mouth-coating, thickness, and astringency with increasing milk solids concentrations of the $\mathrm{C} 1$ and $\mathrm{C} 2$ yogurts. Mean hedonic scores for color, appearance, and texture/mouth-feel were greater than hedonic scores for aroma, flavor/taste, and overall liking. This research identified the sensory qualities that need further development and demonstrated the importance of early-stage consumer acceptance research for directing new product development.

Key words: carrot juice yogurt, probiotic, descriptive analysis, consumer acceptance

\section{INTRODUCTION}

In recent years, consumer interest in foods that claim health benefits beyond basic nutritional properties

Received October 18, 2012.

Accepted March 23, 2013

${ }^{1}$ Corresponding author: Margaret.Cliff@agr.gc.ca has increased dramatically (Hekmat and Reid, 2006). Probiotics are one such product and are defined by the FAO/WHO (2002) as "live microorganisms which, when administered in adequate amounts, confer health benefits on the host." Yogurt is one of the most widely accepted and consumed probiotic food products, and the ingestion of certain lactic acid bacteria (LAB) used to produce yogurt is reported to benefit the health of humans (Shortt, 1999; FAO/WHO, 2006; Giraffa et al., 2010). The probiotic activity of specific LAB has been emphasized (Prasad et al., 1998; Shortt, 1999), with the most widely used being Lactobacillus and Bifidobacterium strains. The vitamin $\mathrm{K}$ produced by LAB may play an important dietary role, along with other components, in the prevention and treatment of osteoporosis (Knapen et al., 1989).

Yogurt is also widely used as a carrier for food components such as fiber, fatty acids, and antioxidants that are believed to improve physiological functions in the body. In addition, carrots are considered to be very nutritious, containing vitamins, minerals, and dietary fiber, as well as flavonoids and carotenoids, all of which are beneficial in preventing diseases (Lampe, 1999).

Currently, no vegetable juice yogurts are available in the North American market. Many types of fruit yogurts have been developed, but the fruit is generally added after fermentation. The goal of this study was to develop a carrot juice yogurt that offers the consumer the combined benefits of LAB and the high $\beta$-carotene content of carrots. The co-fermentation of the vegetable and milk has the potential to create a novel product with unique sensory properties.

Previous research on the sensory quality of carrot yogurt was conducted using a single quality rating for each of flavor, texture, and appearance (Aly et al., 2004). Such a rating system fails to identify the sensory attributes that need to be optimized during product development.

Because taste is one of the most important attributes of functional food (Urala and Lähteenmäki, 2004), it is important to ensure sensory and taste quality during the development of a new yogurt. Flavor acceptability and price (Moskowitz and Chandler, 1978) and taste 
and health claims (Vickers, 1993) have also been shown to have the largest influence on buying intent of yogurt. Although some researchers (Behrens et al., 2007) have observed an influence of health or nutritional claims on acceptability of soy milk beverages, others working with fat-free yogurts have not (Kähkönen et al., 1997).

Incorporating the "voice of the consumer" in the early stages of product development has been demonstrated to play an important role in the ultimate success of a product (van Kleef et al., 2005). Consumers expect plain yogurt to have certain sensory attributes, including creaminess, softness or thickness, and acidity (Ares et al., 2008). Therefore, this research coupled descriptive analysis with consumer acceptance research to allow consumer input to direct development of carrot juice yogurts for the marketplace. Data collection was conducted in 2 locations to access regional and ethnic (Asian versus non-Asian) differences that may exist among consumers between eastern and western Canada (Cliff et al., 1999).

\section{MATERIALS AND METHODS}

\section{Yogurt Preparation}

All yogurt treatments were prepared at the Atlantic Food and Horticulture Research Centre (Kentville, NS, Canada). Yogurts were made with 2 freeze-dried starter cultures from Lyo-San Inc. (Lachute, QC, Canada). Culture 1 (C1) contained bacterial cultures typically used by industry (Lactobacillus bulgaricus, Streptococcus thermophilus, and Lactobacillus acidophilus) with only one probiotic species (L. acidophilus), as well as skim milk powder, sucrose, and ascorbic acid. Culture 2 (C2) contained 3 probiotic species: Lactobacillus casei, Bifidobacterium longum, and L. acidophilus, along with Lactobacillus delbrueckii ssp. bulgaricus and $S$. thermophilus.

Carrot juice was prepared from fresh carrots using a juicer (model 67600 Juice Extractor, Hamilton Beach, Washington, NC) and pasteurizing the juice at $85^{\circ} \mathrm{C}$ for 30 min. Four levels of carrot juice $(8,16,24$, and $32 \%$ ) were tested. Two yogurt firmness levels were evaluated: regular and firm made with 45 and $90 \mathrm{~g} / \mathrm{L}$ of skim milk powder added to $2 \%$ milk, respectively. All yogurts were prepared with $3.5 \mathrm{~g} / \mathrm{L}$ gelatin (Knox, Associated Brands Inc., Toronto, ON, Canada).

Samples for sensory testing were prepared using a split-plot design. In this design, yogurt firmness (2 levels) was the main plot and carrot juice level (4 levels) was the sub-plot. Six replicate batches of each experimental treatment were prepared, 1 batch per day, for each of 6 sensory sessions for each of 2 cultures (C1 and C2). Samples were allocated to sensory panelists using the design described below in the sensory assessment section. Replicate batches of carrot juice by firmness levels were prepared for the consumer tests (Wolfville, NS; Vancouver, BC) and samples assigned to consumers using designs described in the consumer acceptance section.

Formulations were heated to $82^{\circ} \mathrm{C}$ for $2 \mathrm{~min}$, cooled to $40^{\circ} \mathrm{C}$ before adding the starter culture $(5 \mathrm{~g} / \mathrm{L})$, and then cooled to $25^{\circ} \mathrm{C}$ for dispensing. Individual servings (90 $\mathrm{mL}$ in $140-\mathrm{mL}$ sealed containers) were prepared for descriptive analysis and consumer evaluations in Wolfville, whereas 1-L portions were packaged in polypropylene containers for consumer evaluations in Vancouver.

Yogurts made with $\mathrm{C} 1$ were incubated at $43^{\circ} \mathrm{C}$ for $4 \mathrm{~h}$ and cooled at $4^{\circ} \mathrm{C}$ for $8 \mathrm{~h}$. Yogurts made with $\mathrm{C} 2$ were placed an incubator (SSTH-28P-0.5 Burnsco Environmental Test Chambers, Arnprior, ON, Canada) at $25^{\circ} \mathrm{C}$, which was programmed to increase in temperature at a rate of $2^{\circ} \mathrm{C} / \mathrm{h}$ for $9 \mathrm{~h}$ to $43^{\circ} \mathrm{C}$. Yogurts were held at $43^{\circ} \mathrm{C}$ for $6 \mathrm{~h}$, cooled within $1 \mathrm{~h}$ to $4^{\circ} \mathrm{C}$, and held at $4^{\circ} \mathrm{C}$ for $8 \mathrm{~h}$. All temperatures were controlled within $\pm 0.1^{\circ} \mathrm{C}$.

Plain yogurts $(0 \%$ carrot juice) were manufactured similarly. Four treatments of plain yogurt $(0 \%$ carrot juice) at both levels of firmness (regular, $45 \mathrm{~g} / \mathrm{L}$ milk solids; firm, $90 \mathrm{~g} / \mathrm{L}$ milk solids) and using both starter cultures $(\mathrm{C} 1, \mathrm{C} 2)$ were prepared in 3 replicate processing batches.

For sensory descriptive testing, all treatments were held at $3^{\circ} \mathrm{C}$ for $18 \mathrm{~h}$ before assessments. For consumer evaluations, samples were subsequently held at 5 to $6^{\circ} \mathrm{C}$ during ground or air and ground delivery to Acadia University (Wolfville, NS, Canada) and University of British Columbia (UBC, Vancouver, BC, Canada), respectively. An electronic (digital) recorder (Hobo H8, Onset Computer Corp., Bourne, MA) was used to verify the desired shipment temperatures. For the UBC assessment, samples were stored at $5^{\circ} \mathrm{C}$ for an additional $48 \mathrm{~h}$ before evaluations.

\section{Sensory Assessment}

Sensory descriptive analysis of the plain and carrot juice yogurts was conducted at the Atlantic Food and Horticulture Research Centre in Kentville using a 6-member trained sensory panel. Panelists had from 4 to $20 \mathrm{yr}$ of experience with descriptive analysis of foods. During the training sessions $(14 \mathrm{~h})$, panelists selected appropriate sensory attributes, defined the sensory terms, established the assessment procedures, selected reference materials, and practiced rating the experimental yogurts. As shown in Table 1, these sensory terms included 2 visual attributes (color intensity before 
and after stirring), 2 flavor/taste attributes (sourness, carrot flavor), and 7 textural attributes (astringency, chalkiness, mouth-coating, thickness, smoothness, creaminess, and graininess). Descriptive adjectives for sourness (citrus/lemon-like, tomato-like, acetic-like) were developed using a consensus process, and reference standards were developed (Table 1).

All sensory test sessions were conducted in an environment-controlled taste-panel room equipped with individual booths and incandescent lighting. Booths were equipped with computers with Compusense five (version 4.2; Compusense Inc., Guelph, ON, Canada). Ratings were conducted on $10-\mathrm{cm}$ line scales and converted to numerical values $(0.0-10.0)$ for statistical analyses.

Panelists evaluated all 8 yogurts (4 carrot juice levels $\times 2$ firmness levels) in 6 sensory sessions for each of $\mathrm{C} 1$ and $\mathrm{C} 2$ samples. Using a Latin square split-plot design, 4 yogurt treatments $(2$ carrot juice levels $\times 2$ firmness levels) were assigned to a panelist at each session.

All 6 possible pairings of carrot juice level were tested 3 times by each panelist after the 6 sessions. Therefore, $\mathrm{n}=72$ for each firmness level, $\mathrm{n}=36$ for each carrot juice level, and $\mathrm{n}=18$ for carrot juice by firmness combinations.

Panelists evaluated all 4 treatments of plain yogurt from each processing batch at 3 successive sessions. The plain yogurts were evaluated using the same descriptive methodology as described above, with the exception of color intensity and carrot flavor. These yogurts were not included in the descriptive analysis of the carrot juice yogurts, so that panelists could specifically develop terms for describing carrot juice yogurts rather than terms that would be appropriate for both plain and carrot juice yogurts.

\section{Consumer Acceptance Assessments}

C1 Yogurts (Wolfville). All sensory testing was conducted in an environment-controlled, taste-panel room at the Centre for Sensory Research of Food at Acadia University (Wolfville, NS, Canada). In total, 56 subjects were recruited from faculty, staff, students, and area residents by e-mail, phone calls, and printed notices. Subjects were volunteers ( $80 \%$ female, $20 \%$ male) who consumed yogurt 1 to 7 times a week and consumed "unsweetened yogurt" approximately 59\% of the time. Ethics approval was obtained from the Acadia University Research Ethics Board; informed consent was obtained from all participants. Subjects were rewarded with an incentive after completing the yogurt evaluations.

Each consumer evaluated 8 yogurt samples $(8,16$, 24 , and $32 \%$ carrot juice $\times 2$ firmness levels) made with $\mathrm{C} 1$ starter culture over 2 sessions using a balanced design for 56 consumers and 8 products (Wakeling and MacFie, 1995). Each consumer also rated a commercial plain unsweetened yogurt (Danone Silhouette O+, Danone Inc., Boucherville, PQ, Canada) before the carrot yogurt samples. All samples were coded with 3-digit random numbers. Consumers provided hedonic ratings for overall liking for the commercial sample and liking of appearance, flavor/taste, and texture/mouth-feel for each of the experimental yogurts on a 9-point hedonic scale, where $1=$ dislike extremely, $5=$ neither dislike nor like, and $9=$ like extremely. Room temperature spring water and unsalted crackers were provided for rinsing the mouth between samples.

Upon completion of the evaluation consumers indicated how often they might consume such a product on a 7-point scale (never, $<1$ time/mo, $1-3$ times/mo, 1-2 times/wk, 3-4 times/wk, 5-6 times/wk, and $\geq 7$ times/ wk), should it become available in the marketplace.

Data were collected using the Compusense five (version 4.2; Compusense Inc.) sensory data collection system. Preliminary examination of the Wolfville data revealed that the yogurts with the lower firmness $(45$ $\mathrm{g} / \mathrm{L}$ skim milk) and the highest carrot juice (32\%) levels were least preferred; therefore, these treatments were not included in the Vancouver study.

C2 Yogurts (Vancouver). Consumer evaluation of C2 yogurts was conducted at UBC, using 144 consumers (72 Asian, 72 non-Asian). Ethics approval was obtained from the UBC Clinical Research Ethics Board and informed consent was obtained from all participants. Participants were recruited from faculty, staff, and students by e-mail, printed notices, and interception. Participation was restricted to people who consumed yogurt at least 6 times per year. Testing took place over a 2-d period until 72 Asian and 72 non-Asian consumers had been recruited.

Consumers first answered a short questionnaire consisting of 3 sociodemographic questions (age, sex, ethnic origin) and 5 additional questions. Consumers checked the appropriate response categories corresponding to their age $(1=18-30 \mathrm{yr}, 2=31-40 \mathrm{yr}, 3=41-50 \mathrm{yr}$, $4=51-60 \mathrm{yr}, 5=\geq 60 \mathrm{yr}$ ), sex (female, male), and ethnic origin of their ancestors [Asian (China, Hong Kong, Japan, Korean, Taiwan), South Asian (Bangladesh, India, Pakistan), Southeast Asian (Cambodia, Indonesia, Malaysia, Philippines, Vietnam), European (e.g., France, Germany, Italy, Russia, UK), West Asian (e.g., Afghanistan, Iran, Iraq), Latin (e.g., Argentina, Brazil, Chile, Mexico, Venezuela), or other (all other countries)]. Categories were based on classifications of ethnicity used in the Canadian Population Health Survey and the Canadian Community Health Survey (Quan et al., 2006). 


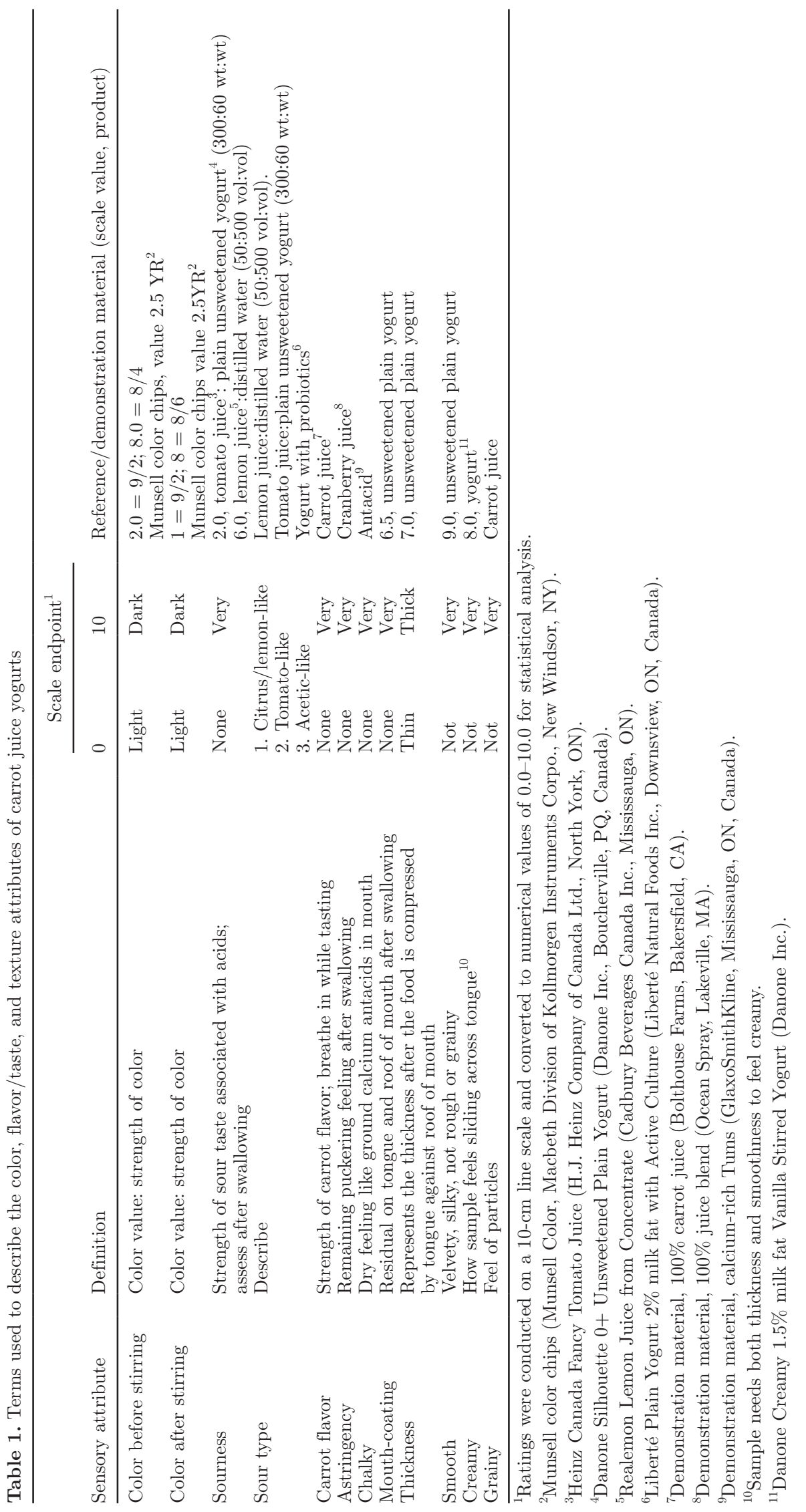


In this research, Asian consumers identified themselves as $84 \%$ Asian, 8\% South Asian, and 8\% Southeast Asian; non-Asian consumers were 78\% European, 11\% West Asian, 7\% Latin, and 4\% other.

The additional questions queried consumers on their frequency of yogurt consumption $(1=1-3$ times $/ \mathrm{mo}$, $2=1-2$ times/wk, $3=3-4$ times/wk, $4=5-6$ times/ wk, $5=\geq 6$ times/wk), knowledge of probiotics and of nutrition, both on 5 -point scales $(1=$ none, $2=$ slight, $3=$ moderate, $4=$ high, $5=$ extreme), awareness of the term prebiotic $(1=$ no, $2=$ yes $)$, and whether they purchased or consumed unsweetened yogurt $(1=$ no, 2 = yes).

After completing the survey questions, consumers evaluated a commercial unsweetened yogurt (Danone Silhouette O+; Danone Inc.) for overall liking using a 9-point hedonic scale. Each consumer then evaluated 3 firm yogurts (90 g/L milk solids) containing 8, 16, and $24 \%$ carrot juice, prepared with culture $\mathrm{C} 2$ for appearance, flavor/taste, texture/mouth-feel, and overall liking using a 9-point hedonic scale. Because the Wolfville assessment had revealed that consumers had particularly disliked the yogurts made with the high carrot juice levels and low firmness levels, these experimental conditions were not included in the Vancouver consumer assessment.

In this design, carrot juice yogurts from 2 processing batches occurred in each tasting position (first, second, third) the same number of times for both groups of consumers. Tasting position was balanced using $3 \times$ 3 Latin squares. Yogurt samples $(25 \mathrm{~mL})$ were served in covered 65-mL (2-oz.) plastic Solo cups (Solo Cup Company, Urbana, IL) coded with 3-digit random numbers. All assessments were conducted on a paper ballots. Participants were given 3 unsalted soda crackers and a glass of room-temperature spring water to rinse their palates between samples.

Upon completion of the evaluation consumers indicated how often they might consume such a product on a 7-point scale (never, $<1$ time/mo, 1-3 times/mo, 1-2 times/wk, 3-4 times/wk, 5-6 times/wk, and $\geq 7$ times/ wk), should it become available in the marketplace.

\section{Statistical Analysis}

Sensory Assessment. Two-factor ANOVA was used to assess the effects of carrot juice level and firmness level and their interaction (carrot juice level $\times$ firmness level), for each bacterial culture (C1, C2). As suggested by Bryan-Jones and Finney (1983) and Chew (1976), mean scores and SEM were reported, rather than using means separation tests. The frequency of use of the sourness descriptors (citrus/lemon-like, tomato-like, acetic-like) were tabulated.
Principal component analysis (PCA) was used to compare the 8 yogurts (2 cultures, 4 carrot juice levels) and evaluate the interrelationship among the attributes. It was conducted using the mean sensory scores for the sensory attributes on the correlation matrix.

Consumer Acceptance Assessment: C1 Yogurts (Wolfville). The main and interactive effects of carrot juice level and firmness level on the hedonic ratings for appearance, flavor/taste, and texture/mouth-feel were examined using ANOVA. Mean scores and SEM were calculated.

Consumer Acceptance Assessment: C2 Yogurts (Vancouver). One-factor ANOVA was used to evaluate the influence of ethnicity (Asian, non-Asian) on the survey variables (yogurt consumption, age, sex, knowledge of probiotics, knowledge of nutrition, aware of prebiotics, and buy/consume unsweetened yogurt). Non-Asian consumers in Vancouver (mean age $=29$ yr) were of a similar age as those who participated in Wolfville (34 yr). However, because non-Asian consumers consumed more yogurt (mean $=3.07 ; 3-4$ times $/$ wk) than Asian consumers (mean $=2.65 ; 2-3$ times/ wk), consumption responses were used as a covariate in ANOVA (analysis of covariance, ANCOVA) to evaluate the influence of ethnicity (Asian, non-Asian), yogurt formulation $(8,16$, and $24 \%$ carrot juice), and their interaction (ethnicity $\times$ carrot juice concentration) for each of the sensory variables. Mean scores and SEM were calculated.

The percentage of consumers who were "likers" and "dislikers/neutral" were determined using hedonic (overall) responses, which were $>5$, and $\leq 5$, respectively, for commercial unsweetened and carrot juice formulations.

All statistical analyses were performed using Genstat for Windows (12th ed., Genstat, VSN International, Hemel Hempstead, UK).

\section{RESULTS AND DISCUSSION}

\section{Sensory Assessment}

$\boldsymbol{A N O V A}$. Color plays an important role in food acceptance. The color intensity of yogurt upon opening the container before stirring is different from that once the product is stirred. Analysis of variance revealed that color intensity was dependent on the combination of carrot juice level and firmness level (Table 2).

The color intensity (before stirring) of the regular firmness C1 yogurts increased across all carrot juice levels. The color intensity of the firm yogurts increased from 8 to $24 \%$ added carrot juice, and changed no further. After stirring, color increased as the level of carrot juice increased; however, the color ratings for yogurts with 8 and $32 \%$ added carrot juice depended on firm- 
Table 2. Means and standard error of the means (SEM) for sensory attributes with a significant firmness by carrot juice interaction ${ }^{1}$

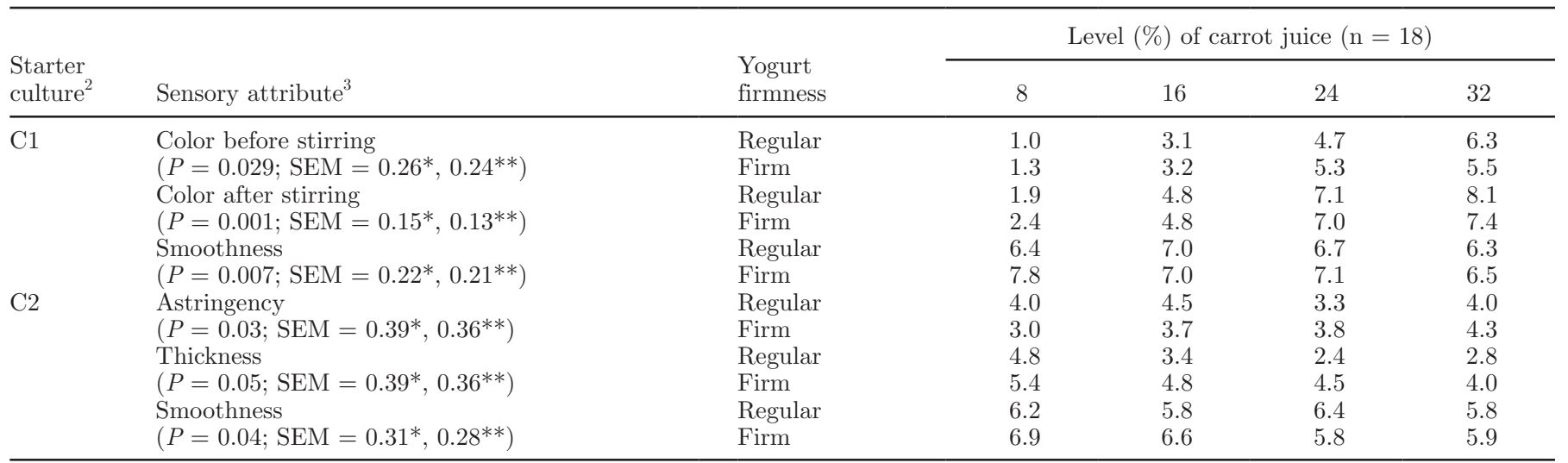

${ }^{1}$ Yogurts were made at 2 firmness levels (regular, $45 \mathrm{~g} / \mathrm{L}$ milk solids; firm, $90 \mathrm{~g} / \mathrm{L}$ milk solids), 4 carrot juice levels, and with 2 starter cultures (C1, C2). Sensory attributes with a significant carrot juice or firmness effects are shown in Table 3.

${ }^{2} \mathrm{C} 1$ : Lactobacillus bulgaricus, Streptococcus thermophilus, Lactobacillus acidophilus; C2: Lactobacillus casei, Bifidobacterium longum, L. acidophilus, Lactobacillus delbrueckii ssp. bulgaricus, and S. thermophilus.

${ }^{3}$ Sensory attributes rated on $10-\mathrm{cm}$ line scales with end-points labeled as $0=$ light and $10=$ dark for color attributes; $0=$ none and $10=$ very for astringency; $0=$ thin and $10=$ thick for thickness; and $0=$ not and $10=$ very for smoothness. All sensory attributes showed a significant carrot juice $\times$ firmness interaction $(\mathrm{n}=18)$.

*SEM for comparing means for carrot juice $\times$ firmness levels; in general, means that differed by 3 times the SEM were significantly different at $P \leq 0.05$.

**SEM for comparing means with the same level of carrot juice.

ness level. The average color before stirring (regular $=3.8$, firm $=3.8$ ) and after stirring (regular $=5.5$, firm $=5.4$ ) did not differ between the 2 firmness levels. Added carrot juice resulted in a linear increase in color intensity for $\mathrm{C} 2$ yogurts before and after stirring (Table 3). Firm C2 yogurts were perceived as being a darker orange color before stirring compared with regular $\mathrm{C} 2$ yogurts; however, firmness had no significant effect on color after stirring.

Panelists described the flavor of this unique yogurt as being dominated by sour taste, carrot aromatics, and chemical feeling of astringency. The characteristic sourness of yogurt was not affected by carrot juice level for $\mathrm{C} 1$; however, when fermented with $\mathrm{C} 2$, the $8 \%$ carrot juice samples were perceived as more sour than samples containing 24 and $32 \%$ carrot juice (Table 3 ). The sweetness of the carrot juice may have reduced the perception of sour at higher additions. Along with intensity ratings for sourness, panelists described the character of the acidity (citrus/lemon-like, tomato-like, or acetic-like). For $\mathrm{C} 1$ and $\mathrm{C} 2$ yogurts, the term "citrus like" was used to describe all carrot juice levels. In contrast, the use of "acetic-like" decreased with increasing carrot juice levels, and the use of "tomatolike" increased with increasing carrot juice levels (data not shown). This suggested that the addition of carrot juice was responsible for the "tomato-like" (herbaceous notes) that were noted as objectionable by some consumers. The control and carrot juice yogurts (C1, C2) did not differ significantly from one another in $\mathrm{pH}$ and titratable acidity, with values ranging from 4.0 to 4.1 and 1.25 to $1.40 \mathrm{~g}$ of lactic acid/100 g, respectively.

The perceived intensity of carrot flavor increased as carrot juice level increased for both $\mathrm{C} 1$ and $\mathrm{C} 2$ yogurts (Table 3). Firmness level had no effect on perceived sourness or carrot flavor (Table 3). The firmness level affected the astringency for $\mathrm{C} 1$ yogurts, with the perceived astringency being higher for the firm yogurts than for the regular yogurts (Table 3). For C2 regular yogurts, those with $24 \%$ carrot juice were perceived as less astringent than those with $16 \%$ carrot juice; however, for the firm $\mathrm{C} 2$ yogurts, those with $8 \%$ carrot juice were perceived as less astringent than those with $32 \%$ carrot juice (Table 2 ).

Panelists evaluated the texture/mouth-feel of the product as thickness, smoothness, and creaminess before swallowing, and the residual perceptions of mouthcoating, chalkiness, and graininess after swallowing. A linear reduction in thickness was observed for $\mathrm{C} 1$ yogurts with increased carrot juice in the formula (Table 3); in addition, firm $\mathrm{C} 1$ yogurts were perceived as thicker. We found an interaction between carrot juice and firmness levels for thickness of $\mathrm{C} 2$ yogurts (Table 2). Firm C2 yogurts gradually became thinner with each successive addition of carrot juice. The thickness of the regular yogurts decreased from 8 to $24 \%$ added carrot juice, and changed no further with each increase in percent carrot juice, except for the highest level.

The interaction between carrot juice and firmness levels for smoothness in $\mathrm{C} 1$ and $\mathrm{C} 2$ yogurts (Table 2) 
Table 3. Means and standard errors of the means (SEM) for sensory attributes with significant firmness or carrot juice effects ${ }^{1}$

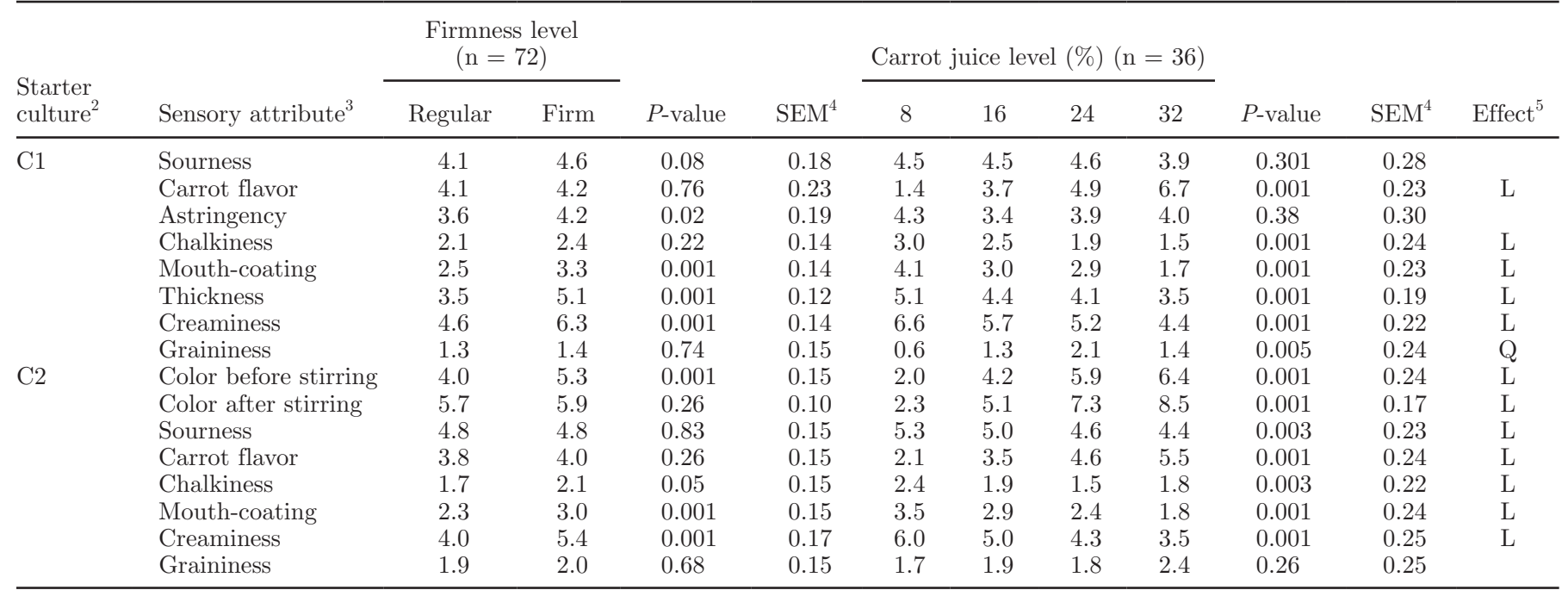

${ }^{1}$ Yogurts were made at 2 firmness levels (regular, $45 \mathrm{~g} / \mathrm{L}$ milk solids; firm, $90 \mathrm{~g} / \mathrm{L}$ milk solids), 4 carrot juice levels, and with 2 starter cultures (C1, C2). Sensory attributes with a significant carrot juice by firmness interactions are shown in Table 2.

${ }^{2} \mathrm{C} 1$ : Lactobacillus bulgaricus, Streptococcus thermophilus, Lactobacillus acidophilus; C2: Lactobacillus casei, Bifidobacterium longum, L. acidophilus, Lactobacillus delbrueckii ssp. bulgaricus, and S. thermophilus.

${ }^{3}$ Sensory attributes rated on 10 -cm line scales with end-points labeled as $0=$ none and $10=$ very for sourness, carrot flavor, astringency, chalkiness, and mouth-coating; $0=$ thin and $10=$ thick for thickness; $0=$ not and $10=$ very for smoothness, creaminess, and graininess; and $0=$ light and $10=$ dark for color attributes.

${ }^{4}$ In general, means that differed by 3 times the SEM were significantly different at $P \leq 0.05$.

${ }^{5} \mathrm{~L}=$ linear effect, $\mathrm{Q}=$ quadratic effect.

was attributed to the magnitude of the change between the regular and firm yogurts for $\mathrm{C} 1(+1.4,0,+0.4$, and +0.2 for yogurts with $8,16,24$, and $32 \%$ carrot juice, respectively). The change in smoothness was slightly different for $\mathrm{C} 2$ yogurts $(+0.7,+0.8,-0.6$, and +0.1 for yogurts with $8,16,24$, and $32 \%$ carrot juice, respectively). Firm yogurts made with 24 and $32 \%$ carrot juice were less smooth than those made with 8 and $16 \%$ carrot juice.

Firmness and carrot juice levels affected the creaminess and mouth-coating feeling of $\mathrm{C} 1$ and $\mathrm{C} 2$ yogurts (Table 3). With increasing carrot juice, creaminess and mouth-coating perception declined linearly in yogurts made with either starter culture. Firm yogurts (made with higher milk solids) were perceived as more mouthcoating and creamier than the regular yogurts (with lower milk solids). Because creaminess is considered an important attribute for consumer acceptance (Ares et al., 2008), an optimum formula for carrot juice yogurt may require fortification with milk solids. Carrot juice yogurts from both cultures (Table 3) were substantially less creamy than the corresponding plain yogurts (Table 4).

Panelists felt that "graininess" best described larger particles left on the tongue after swallowing and "chalkiness" described finer residual calcium-like particles. Graininess did not differ significantly among treatments for yogurts made with C2 (Table 3). However, panelists perceived $\mathrm{C} 1$ yogurt made with $8 \%$ carrot juice to be less grainy than other $\mathrm{C} 1$ yogurts. The level of added carrot juice affected the chalkiness of $\mathrm{C} 1$ and $\mathrm{C} 2$ yogurts (Table 3). Firmness level had no significant effect on the chalkiness of $\mathrm{C} 1$ yogurts, yet firm yogurts made with $\mathrm{C} 2$ samples were perceived as slightly chalkier than regular samples.

$\boldsymbol{P C A}$. The PCA allowed for a direct simultaneous comparison of the yogurt cultures (Figure 1) and an assessment of the interrelationships among the attributes. In the first 2 dimensions, PCA explained $88.8 \%$ of the variation with principal components (PC) I and PC II accounting for 68.2 and $20.6 \%$, respectively.

Principal component I was most heavily loaded in the positive direction with graininess and in the negative direction with smoothness, thickness, creaminess, chalkiness, and mouth-coating. Several sensory attributes were highly correlated as indicated by the relatively small angles among the vectors. Creaminess and thickness were most highly positively correlated $(\mathrm{r}=0.98$, $P \leq 0.001$ ), suggesting that these terms may be tracking the same or similar underlining characteristic(s). This was consistent with the fact that panelists felt that creamy yogurts should be both thick and smooth (Table 1). The other highly positively correlated attributes were tracking covarying characteristics, as 
Table 4. Sensory means and standard errors of the means (SEM) for plain yogurt made with C1 and C2 starter cultures ${ }^{1}$

\begin{tabular}{lcccc}
\hline & \multicolumn{2}{c}{ Starter culture $(\mathrm{n}=18)$} & & \\
\cline { 2 - 3 } Sensory attribute $^{2}$ & $\mathrm{C} 1$ & $\mathrm{C} 2$ & P-value & SEM $^{3}$ \\
\hline Sourness & 3.0 & 5.2 & 0.001 & 0.27 \\
Astringency & 1.9 & 3.0 & 0.04 & 0.37 \\
Chalkiness & 2.4 & 2.8 & 0.30 & 0.27 \\
Mouth-coating & 3.3 & 3.8 & 0.21 & 0.27 \\
Thickness & 6.1 & 6.3 & 0.52 & 0.25 \\
Smoothness & 8.6 & 8.3 & 0.28 & 0.16 \\
Creaminess & 7.3 & 7.7 & 0.34 & 0.25 \\
Graininess & 0.8 & 0.6 & 0.03 & 0.06 \\
\hline
\end{tabular}

${ }^{1} \mathrm{C} 1$ : Lactobacillus bulgaricus, Streptococcus thermophilus, Lactobacillus acidophilus; C2: Lactobacillus casei, Bifidobacterium longum, L. acidophilus, Lactobacillus delbrueckii ssp. bulgaricus, and S. thermophilus.

${ }^{2}$ Sensory attributes rated on $10-\mathrm{cm}$ line scales with end-points labeled as $0=$ 'none' and $10=$ 'very' for sourness, astringency, chalkiness, and mouth-coating, and $0=$ 'thin' and $10=$ 'thick' for thickness, and $0=$ 'not' and $10=$ 'very' for smoothness, creaminess and graininess, with $\mathrm{n}=18$.

${ }^{3}$ In general, means that differed by 3 times the SEM were significantly different at $P \leq 0.05$.

indicated by the lower correlation coefficients. All 5 attributes (smoothness, thickness, creaminess, chalkiness, and mouth coating) successfully tracked textural changes in the yogurts with incremental additions of carrot juice. These intercorrelations included creaminess versus smoothness $(\mathrm{r}=0.91, P \leq 0.001)$, thickness versus smoothness $(\mathrm{r}=0.91, P \leq 0.001)$, chalkiness versus mouth-coating $(\mathrm{r}=0.90, P \leq 0.001)$, creaminess versus mouth-coating $(\mathrm{r}=0.87, P=0.001)$, creaminess versus chalkiness $(\mathrm{r}=0.84, P=0.002)$, thickness versus mouth-coating $(\mathrm{r}=0.82, P=0.003)$, and thickness versus chalkiness $(\mathrm{r}=0.82, P=0.004)$.

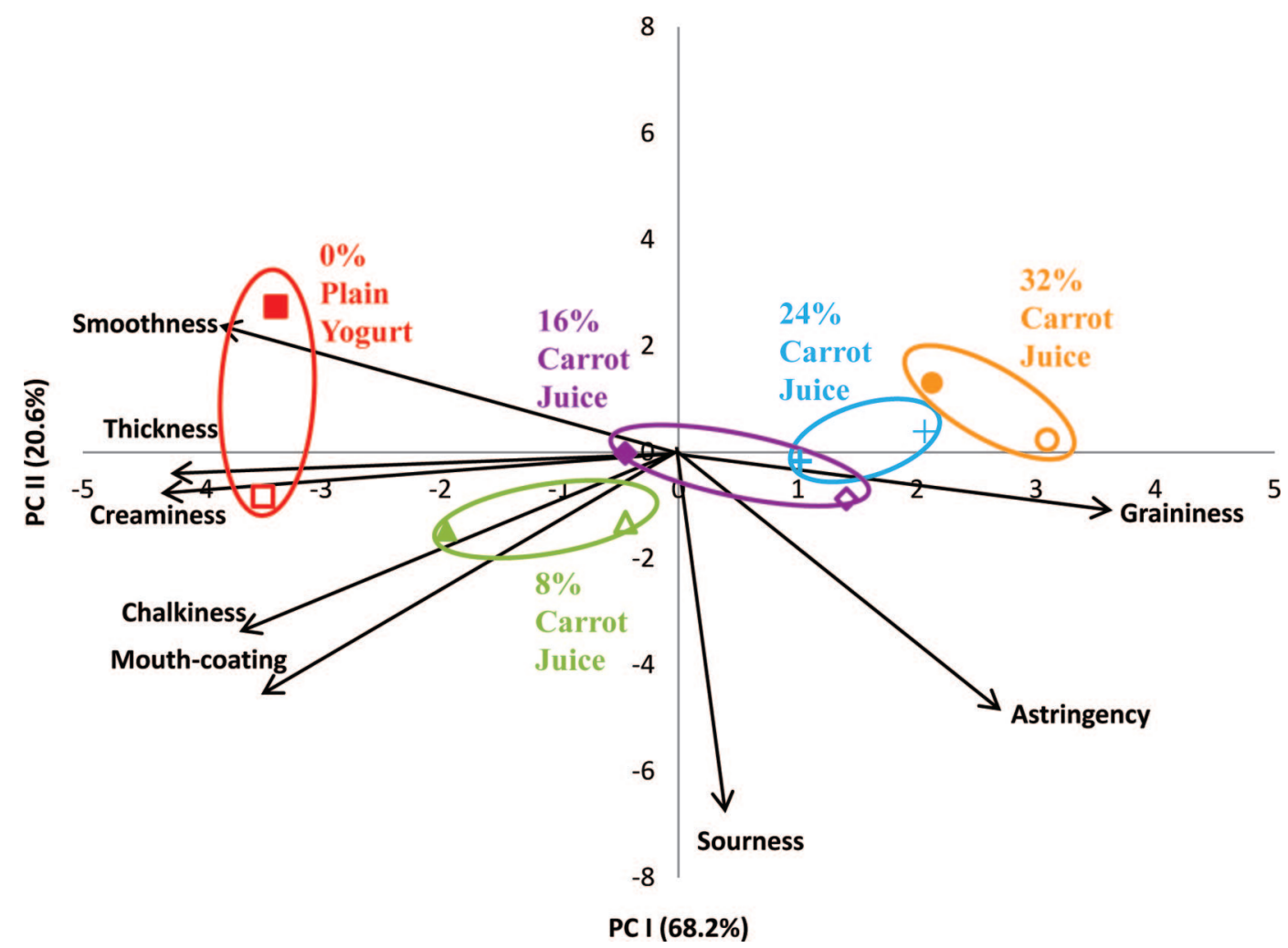

Figure 1. Principal component $(\mathrm{PC})$ analysis for mean $(\mathrm{n}=36)$ sensory scores of 8 yogurts made with 4 levels of carrot juice. Solid and open data points correspond to starter cultures C1 and C2, respectively; C1: Lactobacillus bulgaricus, Streptococcus thermophilus, Lactobacillus acidophilus; C2: Lactobacillus casei, Bifidobacterium longum, L. acidophilus, Lactobacillus delbrueckii ssp. bulgaricus, and S. thermophilus. Color version available in the online PDF. 
The vectors oriented at $180^{\circ}$ from one another indicated that several sensory attributes were inversely or negatively related to one another. These intercorrelations included creaminess versus graininess $(\mathrm{r}=-0.84$, $P=0.002)$, graininess versus smoothness $(\mathrm{r}=-0.79$, $P=0.006)$, graininess versus thickness $(\mathrm{r}=-0.78$, $P=0.008)$, graininess versus chalkiness $(\mathrm{r}=-0.74$, $P=0.014)$, and graininess versus mouth-coating ( $\mathrm{r}$ $=-0.67, P=0.034)$. Thickness and creaminess had similar weight on PC I; smoothness and chalkiness had similar weight on PC I.

Principal component II was most heavily loaded in the negative direction with sourness and astringency. No attributes loaded on PC II in the positive direction. Sourness had more weight on PC II compared with astringency.

When the samples were plotted along PC I and PC II, a systematic progression of carrot juice concentration was observed along PC I from left $(0 \%)$ to right $(32 \%)$. As carrot juice concentration increased, the yogurts became grainier and less smooth, thick, creamy, chalky, and mouth-coating. At each carrot juice level, the $\mathrm{C} 1$ culture was located to the left of the $\mathrm{C} 2$ culture, suggesting that the $\mathrm{C} 1$ culture produced yogurts that were less grainy than the $\mathrm{C} 2$ yogurts, possibly because of the different lactic acid bacteria in $\mathrm{C} 1$ and $\mathrm{C} 2$ and the different amount of exopolysaccharides produced by L. bulgaricus (Pailin et al. 2001).

\section{Consumer Acceptance Assessments}

C1 Yogurts (Wolfville). Although the amount of added carrot juice significantly influenced the sensory attributes of the yogurts (above) made with $\mathrm{C} 1$, the level of carrot juice had no significant effect on the consumers' hedonic ratings for the appearance and flavor of C1 yogurts (Table 5). Firmness level (fortification with milk solids) improved the hedonic scores for appearance (Table 5).

Consumer liking of the $\mathrm{C} 1$ yogurts' texture/mouthfeel was an interesting interaction between firmness level and carrot juice level (Table 5). All 4 levels of carrot juice yogurt made at the high firmness level and the 8 and $16 \%$ carrot juice regular firmness yogurts had higher hedonic ratings than the regular firmness yogurts with 24 or $32 \%$ carrot juice (Table 5 ). In general, higher firmness yogurts had improved hedonic scores for texture/mouth-feel (regular $=6.2$, firm $=7.0 ; P=$ 0.011 , SEM $=0.10$ ). We believe that the loss of creaminess, thickness, and the mouth-coating at the higher levels of carrot juice in the regular yogurts (Table 2) reduced consumer liking of the texture/mouth-feel. As previously reported, consumers have certain expectations for the sensory creaminess and softness or thickness of yogurt (Ares et al., 2008).

Most encouraging was the consumers' interest in the products should they become available: approximately

Table 5. Mean hedonic score (appearance, flavor/taste, texture/mouth-feel) and standard error of the means (SEM) for regular (45 g/L milk solids) and firm (90 g/L milk solids) yogurts, made with 4 levels of carrot juice and $\mathrm{C}^{1}{ }^{\text {starter culture. }}$

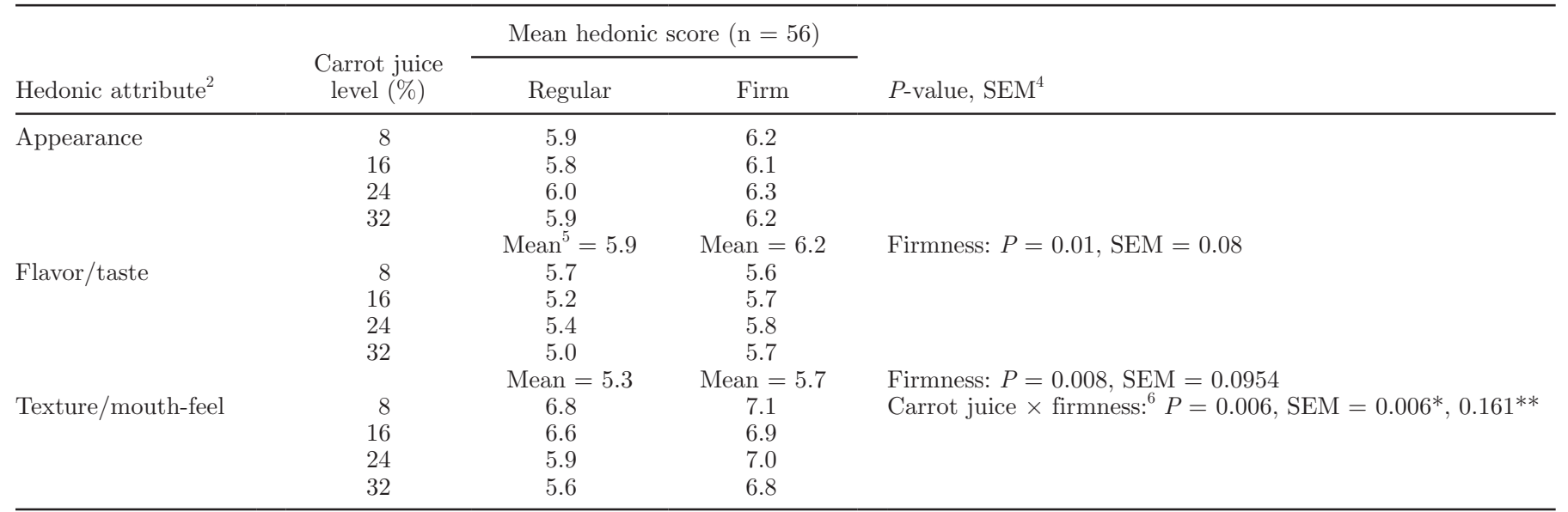

\footnotetext{
${ }^{1}$ Lactobacillus bulgaricus, Streptococcus thermophilus, Lactobacillus acidophilus.

${ }^{2}$ Hedonic attributes rated on 9 -point scales where $1=$ dislike extremely and $9=$ like extremely; $\mathrm{n}=56$ for each carrot juice $\times$ firmness combination, $\mathrm{n}=112$ for each carrot juice level, and $\mathrm{n}=224$ for each firmness level. Consumer assessments were conducted in Wolfville, Nova Scotia.

${ }^{4}$ In general, means that differed by 3 times the SEM were significantly different at $P \leq 0.05$.

${ }^{5}$ Mean value averaged across carrot juice levels.

${ }^{6}$ Significant interaction.

*SEM for comparing means for carrot juice $\times$ firmness levels; means that differed by 3 times the SEM were significantly different at $P \leq 0.05$.

**SEM for comparing means with the same level of carrot juice.
} 
$45 \%$ of consumers indicated that they would buy the product 1 to 3 times/mo and an additional $30 \%$ of consumers indicating that they would consider buying such a product weekly. Considering this was the first exposure, it demonstrated interest in the product. Consumers perceived the product as healthy and versatile, and identified that they would use it for a healthy breakfast, snack or dip, as a cooking ingredient, or as a substitute for sour cream. Although hedonic ratings may seem somewhat low for a new product introduction (appearance $=6.1$, flavor $/$ taste $=5.5$, texture/ mouth-feel $=6.6$ ), they were favorable compared with the hedonic rating for plain yogurt that is currently marketed (overall liking $=5.8$ ). Such scores are very similar to those reported for other novel yogurts (Sanz et al., 2008). Consumers may give lower hedonic ratings to unsweetened yogurt eaten "as is" because such yogurt would frequently be consumed with other foods such as fruit and cereals. Luckow et al. (2006) showed that repeated exposure and health information have positive influences on overall liking, and Jaeger and MacFie (2001) noted that initial product attributes (taste and texture) are simply the signal for the longterm health benefits.

C2 Yogurts (Vancouver). Asian and non-Asian consumers did not differ in their knowledge of probiotics or nutrition, or in their awareness of the term "prebiotics" (data not shown). Although the 2 groups differed $(P \leq 0.05)$ slightly in age $($ Asian $=22$ yr; nonAsian $=29$ yr) and sex (Asian: $82 \%$ female, $18 \%$ male; non-Asian: $60 \%$ female, $40 \%$ male), consumption of yogurt was lower for the Asian consumers (mean = 2.65, 2-3 times/wk) than for the non-Asian consumers (mean $=3.07,3-4$ times $/$ wk). This was consistent with the lower frequency of purchase of unsweetened yogurts by Asian consumers (43\%) compared with their nonAsian $(72 \%)$ counterparts.

The ANCOVA revealed that hedonic scores for yogurt formulations $(8,16$, and $24 \%$ carrot juice) differed significantly for color $(P \leq 0.001)$, appearance $(P \leq$ $0.001)$, and aroma $(P=0.007$; Table 6$)$. We observed a systematic decrease in hedonic mean scores with increasing carrot juice concentration, suggesting that the carrot juice directly or indirectly introduced constituents that were undesirable and distasteful.

The ANCOVA revealed that consumers' ethnicity did not significantly influence the hedonic scores for color, appearance, aroma, and texture/mouth-feel (i.e., means scores were identical for the 2 consumer groups), but did influence the flavor/taste $(P=0.09)$ and overall hedonic $(P=0.007)$ responses. Non-Asian consumers had higher hedonic flavor/taste responses (mean $=4.6-5.3)$ compared with Asian consumers (mean $=$ 4.4-4.5), as well as higher overall responses (non-Asian
$=4.8-5.3 ;$ Asian $=4.5-4.9)$. However, these differences represent the mean response across all yogurt carrot juice concentrations, and the actual pattern varied somewhat with ethnicity $\times$ carrot juice concentration $(P=0.007$; Table 6$)$. The mean responses for the nonAsian consumers declined linearly with increasing carrot juice concentration for flavor/taste $(P=0.094)$ and overall liking $(P=0.007)$. For these consumers, plain unsweetened yogurt was liked (mean $=5.8$ ) more than any of the carrot juice yogurts (mean $=4.8-5.3$; Table $6)$. These scores are centrally located on the 9-point hedonic scale, reflecting products that were "neither liked nor disliked."

In contrast, a slight optimum hedonic response was observed for flavor/taste and overall liking for Asian consumers; however, this should be interpreted with caution, given that consumers scored carrot juice yogurts below the mid-point on the 9-point scale ("disliker"), indicating that these carrot juice yogurts were disliked. This apparent optimum for Asian consumers may be attributed, in part, to the pleasant (attractive) visual characteristics of the yogurts because their hedonic scores for color and appearance were slightly higher than those of non-Asian counterparts (data not shown). As noted above, these optima should be interpreted with caution because all formulations had hedonic scores $<5.0$ by Asian consumers. However, consumers also noted that they might consume these yogurts if they were sweetened.

Such anecdotal comments are consistent with research by Prescott et al. (1992), who reported that Asian consumers had lower hedonic ratings for highsour foods than non-Asian (Australian) consumers, and Druz and Baldwin (1982), who reported that Korean consumers preferred sweeter tomato and apple juices than did non-Asian consumers. However, Prescott et al. (1997) also point out that cross-cultural differences exist not only in the hedonic rating of the sensory attributes but also in the relative importance of several attributes in rating overall liking. Such differences are believed to be particularly important for optimizing the formulation of a product (Prescott et al., 1997).

For all consumers, color and appearance scores were substantially higher than the aroma and flavor/taste scores (Table 6), suggesting that the carrot juice yogurts require additional improvement or product development before release into the commercial marketplace. Anecdotal comments from consumers suggested that the low hedonic responses for the carrot juice yogurts were attributed to the vegetative aromas or flavors and sour or lactic notes.

Vancouver consumers indicated that they would consume the carrot yogurt less frequently than their Wolfville counterparts. Approximately 8 to $11 \%$ of 
Table 6. Mean hedonic score (color, appearance, aroma, flavor/taste, texture/mouth-feel, overall liking) and standard error of the means (SEM) for firm (90 g/L milk solids) yogurts, made with different levels of carrot juice and starter culture $\mathrm{C} 2^{1}$

\begin{tabular}{|c|c|c|c|c|}
\hline \multirow[b]{2}{*}{ Hedonic attribute $^{2}$} & \multirow[b]{2}{*}{$\begin{array}{c}\text { Carrot juice } \\
\text { level }(\%)\end{array}$} & \multicolumn{3}{|c|}{ Mean hedonic score } \\
\hline & & $\begin{array}{l}\text { All consumers }{ }^{3} \\
\quad(\mathrm{n}=144)\end{array}$ & $\begin{array}{l}\text { Asian consumers } \\
\quad(\mathrm{n}=72)\end{array}$ & $\begin{array}{c}\text { Non-Asian } \\
\text { consumers } \\
(\mathrm{n}=72)\end{array}$ \\
\hline \multirow[t]{3}{*}{ Color } & 8 & 6.6 & $\mathrm{NA}^{4}$ & NA \\
\hline & 16 & 6.4 & NA & NA \\
\hline & 24 & 5.5 & NA & NA \\
\hline \multirow{3}{*}{ Appearance } & 8 & 6.6 & NA & NA \\
\hline & 16 & 6.4 & NA & NA \\
\hline & 24 & 5.9 & NA & NA \\
\hline \multirow{3}{*}{ Aroma } & 8 & 5.6 & NA & NA \\
\hline & 16 & 5.5 & $\mathrm{NA}$ & NA \\
\hline & 24 & 5.3 & NA & NA \\
\hline Flavor/taste & 8 & NA & 4.4 & 5.3 \\
\hline \multirow{2}{*}{$\left(P=0.09,{ }^{5} \mathrm{SEM}=0.10^{*}, 0.15^{* *}\right)$} & 16 & NA & 4.5 & 4.8 \\
\hline & 24 & NA & 4.4 & 4.6 \\
\hline \multirow[t]{3}{*}{ Texture/mouth-feel } & 8 & 6.2 & $\mathrm{NA}$ & $\mathrm{NA}$ \\
\hline & 16 & 6.3 & NA & NA \\
\hline & 24 & 6.1 & NA & NA \\
\hline Overall liking & 8 & NA & 4.7 & 5.3 \\
\hline \multirow[t]{2}{*}{$\left(P=0.007\right.$, SEM $\left.=0.15^{*}, 0.19^{* *}\right)$} & 16 & $\mathrm{NA}$ & 4.9 & 5.0 \\
\hline & 24 & NA & 4.5 & 4.8 \\
\hline
\end{tabular}

${ }^{1}$ Lactobacillus casei, Bifidobacterium longum, Lactobacillus acidophilus, Lactobacillus delbrueckii ssp. bulgaricus, and Streptococcus thermophilus.

${ }^{2}$ Hedonic attributes rated on 9 -point scales where $1=$ dislike extremely and $9=$ like extremely. Consumer assessments were conducted in Vancouver, British Columbia $(\mathrm{n}=144)$.

${ }^{3}$ Mean values averaged across all consumers (Asian, non-Asian) because ethnicity was nonsignificant.

${ }^{4} \mathrm{NA}=$ not applicable as ethnicity was not significant.

${ }^{5}$ Probability values $0.05 \leq P \leq 0.10$ reflect possible trends only.

*SEM for comparing means for carrot juice levels.

**SEM for comparing means of different ethnicities.

consumers indicated that they would consume it 1 to 2 times/wk, another 19 to $23 \%$ of consumers indicated they would consume it 1 to 3 times/mo, and more than $60 \%$ of consumers indicated that they would consume it $<1$ time/mo or never. The Vancouver consumers may have been slightly less accepting of an unsweetened carrot juice yogurt than the Wolfville consumers; however, the $\mathrm{C} 2$ yogurts (evaluated in Vancouver) were more astringent, sour, and grainy than the $\mathrm{C} 1$ yogurts (assessed in Wolfville; Figure 1). Although consumers in Wolfville and Vancouver evaluated yogurts prepared with different cultures, mean scores for the commercial plain yogurt (Danone Silhouette O+; Danone Inc.) evaluated initially $($ Kentville $=5.8$, Vancouver $=5.7$ ) indicated that the consumer groups were scoring similarly.

Such findings were consistent with Harper et al. (1991), who identified that many plain yogurts were simply too sour for many consumers. They also reported that there was no relationship between either the sensory or the analytical measurement and overall liking of plain yogurt. Vickers et al. (2001) reported that a lower-than-optimum sweetness level for yogurt was more detrimental to taste ratings than higher-thanoptimum sweetness levels. In contrast, over-sweetened yogurt was detrimental to consumption compared with under-sweetened yogurt (Vickers et al., 2001). Other researchers (Allgeyer et al., 2010) have reported that intermediate sweetness levels were optimal in yogurt drinks with prebiotics and probiotic cultures. In contrast, Lähteenmäki and Tuorila (1994) found that sweet strawberry-flavored yogurts were liked less by consumers who believed that sugar was harmful to their health.

Results were also consistent with the fact that the Asian consumers in this study not only consumed less yogurt but also selected a different type and style of yogurt in the marketplace. In this research, $44 \%$ of Asian consumers liked the commercial plain yogurt compared with $65 \%$ of non-Asian consumers. This difference was believed reflected in the overall hedonic responses to the carrot juice yogurts, with $37 \%$ of Asian consumers scoring the yogurts above the mid-point on the 9-point scale ("likers"), compared with $45 \%$ of non-Asian consumers (data not shown). This suggested that no 
ideal product exists for all consumers but rather that different formulations would be suitable for different sub-groups of consumers.

Such differences between Asian and non-Asian consumers may also be explained by differences in food choice motives. Pohjanheimo and Sandell (2009) demonstrated that consumers who valued a yogurt's natural content and health perceived sourness, thickness, and genuine yogurt flavor as more pleasant, whereas consumers who valued convenience, price, mood, and familiarity evaluated sweetness and smoothness as more pleasant. Although product preferences and choice may differ among cultural groups (e.g., French versus Vietnamese), Tu et al. (2007) showed that the product maps of the sensory characteristics do not differ for soy yogurts. This verifies the importance of the use of descriptive analysis in the developmental phase of product development to fully understand product characteristics before release of new products into the marketplace.

Although some consumers in this study seem to be more receptive to new or different types of yogurt, a full appreciation of consumer acceptance would need to be captured using such tools as focus groups and preference mapping. In this research, non-Asian consumers were primarily European $(78 \%)$ in origin; however, a small fraction (11\%) was West Asian (Afghanistan, Iran, Iraq). Interestingly, these West Asian consumers had a much higher hedonic score for the plain yogurt (mean $=6.9)$ but their scores for the carrot juice yogurts were considerably lower $(3.9-4.6 ; \mathrm{n}=8)$ than those from European consumers (4.7-5.7; n $=56)$. These scores reflect possible differences in neophobia (Henriques et al., 2009; Meiselman et al., 2010) but also illustrate the difficulty of identifying sub-groups of potential consumers for new food products.

\section{CONCLUSIONS}

This research successfully profiled the sensory characteristics of carrot juice yogurt using descriptive analysis with a trained panel. We characterized the magnitude of the color, aroma, flavor/taste, and textural attributes of yogurts prepared with 4 carrot juice levels, 2 firmness levels, and 2 starter cultures. These sensory differences allowed interpretation of the hedonic responses and provided insight into appropriateness of the formulations. Careful consideration needs to be given to the aroma/flavor, acidity, and balance/harmony of the yogurts before release into the marketplace. A focus group might be useful in identifying the most appropriate sweetener(s) for a "sweetened" or reformulated carrot juice yogurt. In addition, special attention should be given to the preferences of the specific sub-groups of consumers for successful development and improvement of carrot juice yogurts.

\section{ACKNOWLEDGMENTS}

The authors thank Sherry Fillmore and Brad Walker at the Atlantic Food and Horticulture Research Centre (Kentville, NS, Canada) for assistance with the statistical analyses.

\section{REFERENCES}

Allgeyer, L. C., M. J. Miller, and S.-Y. Lee. 2010. Drivers of liking for yogurt drinks with prebiotics and probiotics. J. Food Sci. 75:S212-S219.

Aly, S. A., E. A. Galal, and N. A. Elewa. 2004. Carrot yoghurt: Sensory, chemical, microbiological properties and consumer acceptance. Pakistan J. Nutr. 3:322-330.

Ares, G., A. Giménez, and A. Gámbaro. 2008. Understanding consumers' perception of conventional and functional yogurts using word association and hard laddering. Food Qual. Prefer. 19:636-643.

Behrens, J. H., N. D. M. Villanueva, and M. A. A. P. da Silva. 2007. Effect of nutrition and health claims on the acceptability of soyamilk beverages. Int. J. Food Sci. Technol. 42:50-56.

Bryan-Jones, J., and D. J. Finney. 1983. On an error in "Instructions to Authors". HortScience 18:279-282.

Chew, V. 1976. Comparing treatment means: A compendium. HortScience 11:348-357.

Cliff, M. A., K. Sanford, and E. Johnston. 1999. Evaluation of hedonic scores and R-indices for visual, flavour and texture preferences of apple cultivars by British Columbian and Nova Scotian consumers. Can. J. Plant Sci. 79:395-399.

Druz, L. L., and R. E. Baldwin. 1982. Taste thresholds and hedonic responses of panels representing three nationalities. J. Food Sci. 47:561-563.

FAO/WHO. 2002. Guidelines for the evaluation of probiotics in food. Joint FAO/WHO Working Group Report on Drafting Guidelines for the Evaluation of Probiotics in Food, London, ON, Canada. Accessed Sep. 24, 2012. ftp://ftp.fao.org/es/esn/food/wgreport2. pdf.

FAO/WHO. 2006. Probiotics in food: Health and nutritional properties and guidelines for evaluation. Food and Nutrition Paper 85. Food and Agricultural Organization, Rome, Italy. Accessed Sep. 24, 2012. ftp://ftp.fao.org/docrep/fao/009/a0512e/a0512e00.pdf.

Giraffa, G., N. Chanishvili, and Y. Widyastuti. 2010. Importance of lactobacilli in food and feed biotechnology. Res. Microbiol. 161:480-487.

Harper, S. J., D. L. Barnes, F. W. Bodyfelt, and M. R. McDaniel. 1991. Sensory ratings of commercial plain yogurts by consumer and descriptive panels. J. Dairy Sci. 74:2927-2935.

Hekmat, S., and G. Reid. 2006. Sensory properties of probiotic yogurt is comparable to standard yogurt. Nutr. Res. 26:163-166.

Henriques, A. S., S. C. King, and H. L. Meiselman. 2009. Consumer segmentation based on food neophobia and its application to product development. Food Qual. Prefer. 20:83-91.

Jaeger, S. R., and H. J. H. MacFie. 2001. The effect of advertising format and means-end information on consumer expectations for apples. Food Qual. Prefer. 12:189-205.

Kähkönen, P., H. Tuorila, and H. Lawless. 1997. Lack of effect of taste and nutrition claims on sensory and hedonic responses to a fat-free yogurt. Food Qual. Prefer. 8:125-130.

Knapen, M. H. J., K. Hamulyák, and C. Vermeer. 1989. The effect of vitamin $\mathrm{K}$ supplementation on circulating osteocalcin (bone Gla protein) and urinary calcium excretion. Ann. Intern. Med. 111:1001-1005.

Lähteenmäki, L., and H. Tuorila. 1994. Attitudes towards sweetness as predictors of liking and use of various sweet foods. Ecol. Food Nutr. 31:161-170. 
Lampe, J. W. 1999. Health effects of vegetables and fruit: Assessing mechanisms of action in human experimental studies. Am. J. Clin. Nutr. 70:475S-490S.

Luckow, T., V. Sheehan, G. Fitzgerald, and C. Delahunty. 2006. Exposure, health information and flavour-masking strategies for improving the sensory quality of probiotic juice. Appetite 47:315-323.

Meiselman, H. L., S. C. King, and M. Gillette. 2010. The demographics of neophobia in a large commercial US sample. Food Qual. Prefer. 21:893-897.

Moskowitz, H. R., and J. W. Chandler. 1978. Consumer perceptions, attitudes and trade-offs regarding flavor and other product characteristics. Food Technol. 32:34-37.

Pailin, T., D. H. Kang, K. Schmidt, and D. Y. C. Fung. 2001. Detection of extracellular bound proteinase in EPS-producing lactic acid bacteria cultures on skim milk agar. Lett. Appl. Microbiol. $33: 45-49$.

Pohjanheimo, T., and M. Sandell. 2009. Explaining the liking for drinking yoghurt: The role of sensory quality, food choice motives, health concern and product information. Int. Dairy J. 19:459-466.

Prasad, J., H. Gill, J. Smart, and P. K. Gopal. 1998. Selection and characterisation of Lactobacillus and Bifidobacterium strains for use as probiotics. Int. Dairy J. 8:993-1002.

Prescott, J., G. A. Bell, R. Gillmore, M. Yoshida, M. O'Sullivan, S. Korac, S. Allen, and K. Yamazaki. 1997. Cross-cultural comparisons of Japanese and Australian responses to manipulations of sweetness in foods. Food Qual. Prefer. 8:45-55.

Prescott, J., D. Laing, G. Bell, M. Yoshida, R. Gillmore, S. Allen, K. Yamazaki, and R. Ishii. 1992. Hedonic responses to taste solutions: A cross-cultural study of Japanese and Australians. Chem. Senses $17: 801-809$.
Quan, H., A. Wong, D. Johnson, and W. A. Ghali. 2006. The public endorses collection of ethnicity information in hospital: Implications for routine data capture in Canadian health systems. Healthc. Policy 1:55-64.

Sanz, T., A. Salvador, A. Jiménez, and S. M. Fiszman. 2008. Yogurt enrichment with functional asparagus fibre. Effect of fibre extraction method on rheological properties, colour, and sensory acceptance. Eur. Food Res. Technol. 227:1515-1521.

Shortt, C. 1999. The probiotic century: Historical and current perspectives. Trends Food Sci. Technol. 10:411-417.

Tu, V. P., D. Valentin, F. Husson, A. Sutan, D. T. Ha, and C. Dacremont. 2007. How does culture affect food perception and description? Contrasting French and Vietnamese panelists on soy-yogurts. Pages 102-110 in SPISE Symposium, HoChiMinh-City, Vietnam.

Urala, N., and L. Lähteenmäki. 2004. Attitudes behind consumers willingness to use functional foods. Food Qual. Prefer. 15:793803.

van Kleef, E., H. C. M. van Trijp, and P. Luning. 2005. Consumer research in the early stages of new product development: A critical review of methods and techniques. Food Qual. Prefer. 16:181-201.

Vickers, Z. 1993. Incorporating tasting into a conjoint analysis of taste, health claim, price and brand for purchasing strawberry yogurt. J. Sens. Stud. 8:341-352.

Vickers, Z., E. Holton, and J. Wang. 2001. Effect of ideal-relative sweetness on yogurt consumption. Food Qual. Prefer. 12:521-526.

Wakeling, I. N., and J. H. MacFie. 1995. Designing consumer trials balanced for first and higher orders of carry-over effect when only a subset of $k$ samples from $t$ may be tested. Food Qual. Prefer. 6:299-308. 